\section{International Scientific Journal Theoretical \& Applied Science}

\author{
p-ISSN: 2308-4944 (print) e-ISSN: 2409-0085 (online) \\ Year: $2018 \quad$ Issue: $01 \quad$ Volume: 57 \\ Published: $30.01 .2018 \quad$ http://T-Science.org
}

SECTION 25. Technologies of materials

for the light and textile industry.
Maxim Andreevich Pavlov graduate student The Kosygin State University of Russia maxpavlov@live.ru

Elena Aleksandrovna Kirsanova doctor of technical Sciences, Professor The Kosygin State University of Russia

Anastasija Vladimirovna Vershinina graduate student

The Kosygin State University of Russia

\title{
PROGRAM COMPLEX ON PROJECTION AND THE CHOICE OF PACKAGES AND MATERIALS OF LIGHT INDUSTRYMATERIALS
}

Abstract: In article, the basic principles of the device of expert system are displayed. Implementation of this system with integration of the database in which information on characteristics and properties of packets of materials relevant today is put is considered. Key features of facet classification are displayed. Application of heuristic receptions for search of new technology solutions of tasks of design of materials and packets is justified. The software complex allowing creating and projecting the new materials exploited in the given conditions according to the specification is developed.

Key words: package of materials, new properties of materials, database, knowledge base, classification, search of new technology solutions.

Language: Russian

Citation: Pavlov MA, Kirsanova EA, Vershinina AV (2018) PROGRAM COMPLEX ON PROJECTION AND THE CHOICE OF PACKAGES AND MATERIALS OF LIGHT INDUSTRYMATERIALS. ISJ Theoretical \& Applied Science, 01 (57): 186-190.

Soi: http://s-o-i.org/1.1/TAS-01-57-33 Doi: crossef https://dx.doi.org/10.15863/TAS.2018.01.57.33

\section{ПРОГРАММНЫЙ КОМПЛЕКС ПО ПРОЕКТИРОВАНИЮ И ВЫБОРУ ПАКЕТОВ И МАТЕРИАЛОВ ЛЕГКОЙ ПРОМЫШЛЕННОСТИ}

Аннотация: Отображень основные принцииь устройства экспертной системьл. Рассмотрена реализаџия данной системы с интеграцией базы данных, в которую заложена информация по характеристикам и свойствам актуальных на сегодняшний день пакетов материалов. Отображены ключевые возможности фасетной классификации. Обосновано применение эвристических приемов для поиска новых технологических решений задач проектирования материалов и пакетов. Разработан комплекс программного обеспечения, позволяющий создавать и проектировать новые материаль, эксплуатируемые в заданных условиях, в соответствии с техническим заданием.

Ключевые слова: пакет материалов, новые свойства материалов, база данных, база знаний, классификация, поиск новых технологических решений.

\section{Введение}

Материалы для одежды с улучшенными эксплуатационными свойствами, устойчивые к износу, различным видам излучения и давлению, легкие и при этом высокопрочные, нужны для развития практически всех отраслей экономики. Количество таких материалов с каждым днем растет, это может быть следствием того, что возросли потребности в новых свойствах материалов. [1, с 49.]. Именно развитие таких показателей позволяет совершенствовать современные технологии, делая их более экономически выгодными и эффективными, а также реализовывать принципиально другие, наилучшие технологические решения.
В настоящее время разрабатываются новые виды материалов, которые могут быть использованы для защиты от холода, от действия различных химических реагентов, или огнестойкие [2, с 26; 3, с.54; 4 с.49].

При проектировании таких материалов и пакетов зачастую используются уже известные технические решения. и ограниченно применяется информация о новых достижениях в смежных областях наук, например, в физическом материаловедении, химии высокомолекулярных соединений, что ограничивает ассортимент получаемых изделий. Следует отметить, что недостаточно проработан вопрос о процессах алгоритмизации в поиске новых технических 


\begin{tabular}{|c|c|c|c|c|c|c|}
\hline Impact Factor: & $\begin{array}{l}\text { ISRA (India) } \\
\text { ISI (Dubai, UAE } \\
\text { GIF (Australia) } \\
\text { JIF }\end{array}$ & $\begin{array}{r}=1.344 \\
=0.829 \\
=0.564 \\
=1.500\end{array}$ & $\begin{array}{l}\text { SIS (USA) } \\
\text { PИНЦ (Russia) } \\
\text { ESJI (KZ) } \\
\text { SJIF (Morocco }\end{array}$ & $\begin{array}{l}=0.912 \\
=0.207 \\
=4.102 \\
=\mathbf{2 . 0 3 1}\end{array}$ & $\begin{array}{l}\text { ICV (Poland) } \\
\text { PIF (India) } \\
\text { IBI (India) }\end{array}$ & $\begin{array}{l}=6.630 \\
=1.940 \\
=4.260\end{array}$ \\
\hline
\end{tabular}

решений этой проблемы. Ученые, и практики, работающие в области конфекционирования материалов в пакеты изделия, в основном опираются на уже существующий технологические процессы изготовления швейных изделий [5, с.54].

Сегодняшний технологический уклад экономики, именуемый «Индустрия 4.0.» требует от промышленных предприятий цифрового проектирования и моделирования технологических процессов, объектов, изделий на всем жизненном цикле от идеи до эксплуатации (применение инженерного программного обеспечения) [6, с.16.].

Для взаимодействия всей доступной на сегодняшний день информации о пакетах материалов и использования полученного опыта при их проектировании, существует потребность в создании комплекса программного обеспечения на основе базы знаний и фасетной классификации. [7, с.62].

\section{Результаты и обсуждение.}

Созданная нами база знаний работает совместно с реализованной поисковой системой, представляющей собой отбор записей, удовлетворяющих условиям поиска, заданным в форме запроса.
Такая интеллектуальная платформа имеет набор механизмов, запуск которых позволяет подобрать оптимальное техническое решение для создания материала, обладающего необходимыми эксплуатационными свойствами.

База знаний - это база данных, которая содержит правила вывода и информацию об опыте и знаниях в определенной области. [8 c.83]

Полноценная база знаний, в отличие от обычной, содержит в себе не только текущую информацию, но и правила, которые позволяют делать выводы об уже имеющихся или дополнительно вводимых фактах и таким образом выполнять семантическую (смысловую) обработку информации.

База знаний по материалам, пакетам и конфекционированию используются в контексте экспертной системы, где с еe помощью представляются навыки и опыт экспертов, специалистов в области легкой промышленности.

Система, в которой реализовано взаимодействие пользователя и эксперта (инженера по знаниям) с базой знаний, можно считать экспертной системой или комплексом программного обеспечения (Рис. 1), помогающего специалисту принимать обоснованные решения.

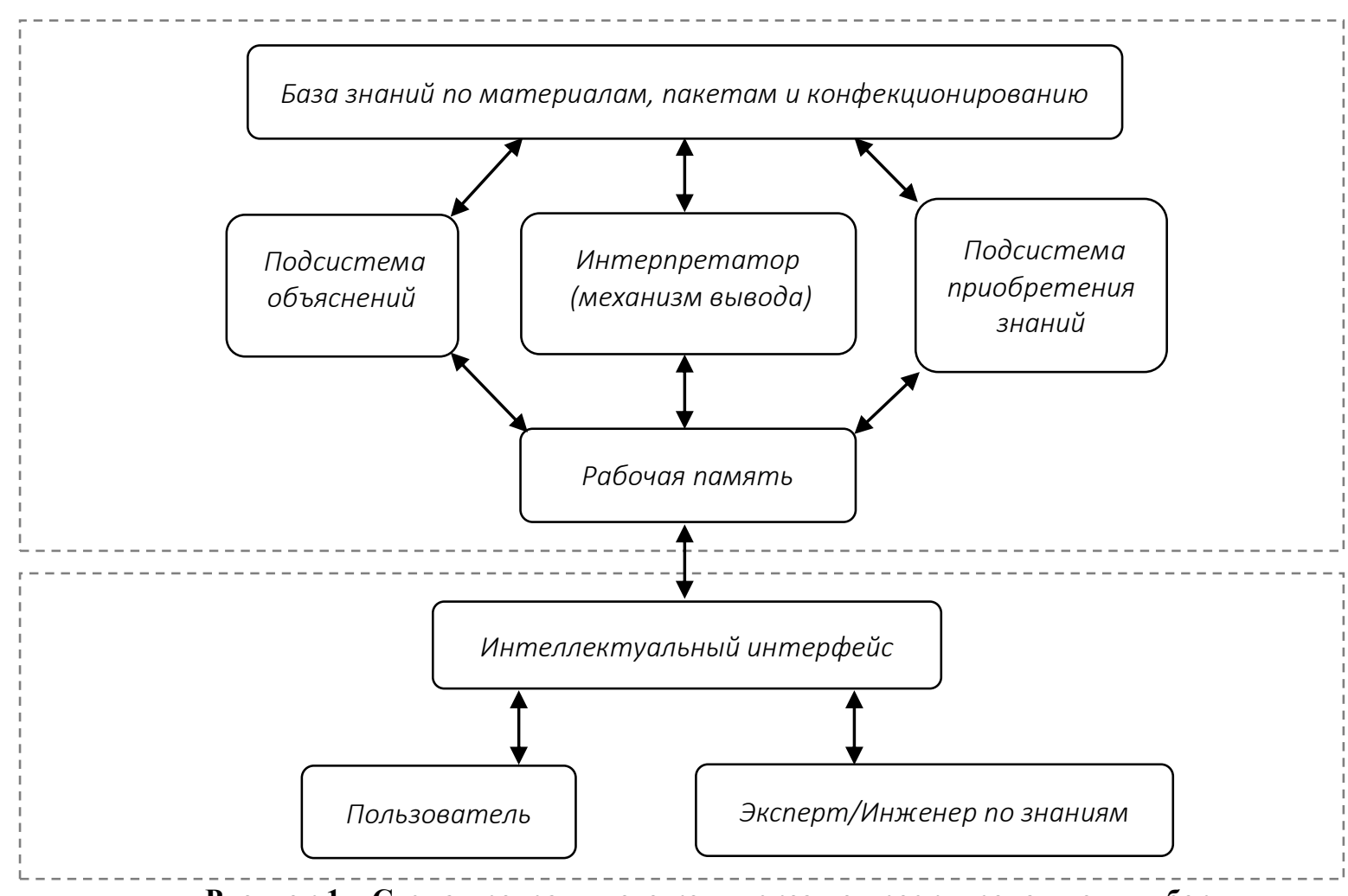

Рисунок 1 - Схема программного комплекса по проектированию и выбору пакетов и материалов легкой промышленности

На схеме отображены следующие элементы: база знаний, включает в себя информацию по материалам, пакетам и конфекционированию; интерпретатор базы знаний - это 


\begin{tabular}{|c|c|c|c|c|c|c|}
\hline Impact Factor: & $\begin{array}{l}\text { ISRA (India) } \\
\text { ISI (Dubai, UAE } \\
\text { GIF (Australia) } \\
\text { JIF }\end{array}$ & $\begin{array}{l}=1.344 \\
=0.829 \\
=0.564 \\
=1.500\end{array}$ & $\begin{array}{l}\text { SIS (USA) } \\
\text { PИНЦ (Russia) } \\
\text { ESJI (KZ) } \\
\text { SJIF (Morocco) }\end{array}$ & $\begin{array}{l}=0.912 \\
=0.207 \\
=4.102 \\
=\mathbf{2 . 0 3 1}\end{array}$ & $\begin{array}{l}\text { ICV (Poland) } \\
\text { PIF (India) } \\
\text { IBI (India) }\end{array}$ & $\begin{array}{l}=6.630 \\
=1.940 \\
=4.260\end{array}$ \\
\hline
\end{tabular}

управляющая система, организующая вывод решения, связь с пользователем, выбор и активация нужного раздела базы знаний;

подсистема приобретения знаний, в которой работа с содержимым и наполнение базы знаний осуществляется посредством «редактора».

подсистема объяснений выдает пользователю информацию о том, почему получены результаты. так как пояснение шагов работы системы является гарантией доверия к полученным результатам, что особенно важно в системах, связанных с принятием решений;

рабочая память хранит результаты предварительных вычислений и выводов, информацию о используемых знаниях о материалах, пакетах и конфекционированиии, актуальные запросы пользователей и т.п.

интеллектуальный интерфейс - подсистема, обеспечивающая общение с пользователем удобным ему способом.

пользователь - специалист из области легкой промышленности, который работает с системой: конфекционер, технолог, конструктор, лекальщик, дизайнер и др.

эксперт-специалист с высокой квалификацией в данной предметной области, обладающий специальными познаниями и навыками решения задач, для которых предназначена система, а также обладающий опытом работы с экспертной системой.

инженер по знаниям -специалист, обладающий методами и способами работы с экспертами, способами извлечения и интерпретации знаний, понимающий технологии экспертных систем.

Для корректной работы программного обеспечения в базу заложена фасетная модель классификации информации и условный формат представления знаний. Такая интерпретация информации в области текстильного материаловедения, вместе с данными о свойствах конкретных пакетов материалов, в совокупности является базой знаний в данной области [9 с.342].

Разработанная фасетная навигация основана на соображении, что пользователи могут искать информацию различными способами. При выборе пакета материала первым запросом является, например, волокнистый состав слоев, а для второго запроса - его способ производства. Таким образом, фасеты составляют множества возможных значений (рис.2).



Рисунок 2 - Фасетная классификация по множеству признаков материалов и пакетов.

Пересечение множеств дает однозначную идентификацию объекта и тем самым получаем фасетную формулу первого уровня (1).

$$
\Pi M 1=\Phi 1, \Phi 2, \Phi 3
$$

где Фі - множество значений признаков фасетной классификации.

В качестве примера рассмотрим задачу совершенствования специальной одежды с целью улучшения теплозащитных свойств. В 
идеализированном случае спецодежда полностью выполняет свою функцию, она является барьером, препятствующим воздействию вредных факторов внешней среды и обеспечивает тепловой баланс тела, сохраняя часть выделяемого тепла и отводя излишки в окружающую среду. Так же, человеком выделяется влага и углекислый газ.

На первом этапе работы спроектированной системы осуществляем выбор климатической зоны, каждой из которой соответствует определенный банк пакетов и изделий с набором критериев: по составу, по способу соединения, по количеству слоев с числовыми характеристиками свойств этих материалов и т.д. Этот банк представляет собой базу данных, в структуру которой также заложена фасетная система классификации. Для экспертов предоставлен вариант «вручную» задать климатические условия эксплуатации одежды, а инженеру по знаниям добавлять новые разработанные пакеты материалов и их свойства.

После определения климатических условий или выбора зоны, система предоставляет выборку в виде списка соответствующих пакетов материалов с их свойствами и характеристиками. Далее из этого списка можно выбрать желаемые параметры эксплуатируемой одежды. Имея выборку пакетов, целью остается определить наиболее подходящие изделия для эксплуатации в заданных условиях. Однако, когда при попытке улучшить одну характеристику или показатель качества технического объекта ухудшается другая его характеристика или показатель. Для устранения таких технических противоречий, используем заложенные в систему приемы разрешения, основанные на анализе большого количества изобретений Г.С. Альтшуллером [11 c.121.]. Каждый из приемов достаточно универсален и отражает наиболее эффективные принципы преобразования объекта.

Ключевым компонентом, в помощи принятия решения специалисту, является алгоритм, обрабатывающий набор заранее заложенных эвристических приемов решения задач в зависимости от запросов пользователя, поэтому выбираются только подходящие пакеты материалов. На данном шаге в созданной системе и базе данных происходит выборка. Методом исключения неудовлетворительных запросов по базе, пакеты сортируются, связи которых в базе. Если их связи не нарушаются, то в исключительном порядке пакеты отображаются на экране пользователя.

К перечисленным выше показателям задача заключается в уменьшении влияния внешней среды, действующей на человека, например, температура, ветер, влага. Одни показатели относятся к спецодежде, а другие к самому человеку. Таким образом, в задаче необходимо минимизировать воздействие метеорологических условий, действующих на человека извне. Если решать задачу известными методами, т.е. увеличить толщину пакета материала с целю увеличения термического сопротивления одежды, то ряд показателей ухудшится. Так увеличится масса и объем спецодежды, ухудшится удобство эксплуатации. повысится сложность устройства и т.д.

Применение эвристических приемов к данной задаче может привести, например, к следующим решениям:

1. Изготовить утеплитель спецодежды разъемными, т.е. в виде утепляющей прокладки.

2. Применить пористые и композитные материалы с различными газообразными наполнителями.

3. Использовать вентиляционные отверстия и воздушные каналы.

4. Использовать

дополнительные источники обогрева различных видов энергии.

Следует отметить, что наилучший итог изобретения получается при использовании нескольких приемов одновременно. Так, например, при решении задачи, заключающейся в реализации возможности регулирования размеров пакетов, при выполнении разного вида работ, вследствие чего изменяется термическое сопротивление одежды и обеспечивается комфортность изделия. Эта задача решается за счет того, что утеплитель расположенный между внешним и внутренним слоями выполнен из растяжимого эластомерного материала, а скрепление всех слоев пакета осуществляется при растяжении внутреннего теплозащитного материала.

\section{Выводы}

Предложенный программный комплекс обеспечивает разработку технического задания на проектирование новых материалов и осуществляет обоснованный выбор материала для одежды с заданными свойствами. Реализация системы предусматривает интеграцию в большинство комплексов проектирования для текстильного и швейного производств.

Использование программного комплекса расширяет ассортимент рекомендуемых материалов и пакетов для конкретных условий в соответствии с возможностями производства.

Применение фасетной классификации в разработанном комплексе обеспечивает гибкость структуры его содержимого. Изменения в содержимом одних фасет не влияет на другие, таким образом, обеспечена приспособляемость классификации к изменяемому характеру поставленных задач. Обеспечена возможность агрегации объектов и выполнение поиска 


\begin{tabular}{l|lr|ll|ll} 
& ISRA (India) & $=\mathbf{1 . 3 4 4}$ & SIS (USA) & $=\mathbf{0 . 9 1 2}$ & ICV (Poland) & $=\mathbf{6 . 6 3 0}$ \\
Impact Factor: & ISI (Dubai, UAE) $=\mathbf{0 . 8 2 9}$ & PUH (Russia) $=\mathbf{0 . 2 0 7}$ & PIF (India) & $=\mathbf{1 . 9 4 0}$ \\
& GIF (Australia) & $=\mathbf{0 . 5 6 4}$ & ESJI (KZ) & $=4.102$ & IBI (India) & $=\mathbf{4 . 2 6 0}$ \\
& JIF & $=\mathbf{1 . 5 0 0}$ & SJIF (Morocco) & $=2.031$ & & \\
\hline
\end{tabular}

информации и подбор параметров по любому

сочетанию фасетов.

\section{References:}

1. Vershinina A.V., Ionova M.H., Kirsanova E.A., Pavlov M.A. (2017) Issledovanie svojstv funkcional"nyh materialov dlja odezhdy raznogo naznachenija// $\mathrm{V}$ sb.: Innovacionnye vnedrenija v oblasti tehnicheskih nauk sbornik nauchnyh trudov po itogam mezhdunarodnoj nauchno-prakticheskoj konferencii. Federal'nyj centr nauki i obrazovanija "Jevensis". 2017. p. 48-50

2. Mishakov V.Ju., Sovetnikov D.A., Pavlov M.A., Kirsanova E.A. (2017) Razrabotka metoda analiza i rascheta jeffektivnogo kojefficienta teploprovodnosti netkanogo teploizoljacionnogo materiala // Theoretical \& Applied Science. 2017. № 7 (51). p. 21-27

3. Kostomarov S.A., Shustov Ju.S., Kurdenkova A.V., Valuev V.S. Byzova E.V. (2017) Razrabotka algoritma ocenki kachestva tkanej special'nogo naznachenija dlja zashhity ot kislot i shhelochej algoritm// Dizajn i tehnologii № 61 (103). - 2017. - p. 53-57

4. V.I. Besshaposhnikova, S.V. Rode, L.A. Lipatova, I.N. Zhagrina, V.I. Lobov (2017) Characteristics of Structure Formation in Temperature-Regulated Textile Materials// Fiber chemistry- №1, 2017. p. 49-52.

5. Zolotceva L.V., Chalenko E.A., Trutneva N.E. (2017) Koncepcija razrabotki metoda proektirovanija verhnej zhenskoj odezhdy na individual'nogo potrebitelja $\mathrm{v}$ uslovijah promyshlennogo proizvodstva. // Dizajn i tehnologii № 59 (101). - 2017. - p. 53-58

6. (2017) Informacionno-analiticheskij otchet. Analiz mirovogo opyta razvitija promyshlennosti i podhodov $\mathrm{k}$ cifrovoj transformacii promyshlennosti gosudarstvchlenov Evrazijskogo jekonomicheskogo sojuza. (Malov A. Ju., Ivanov M. O.) Moskva, 2017.

Available:

http://www.eurasiancommission.org/ru/act/pro m_i_agroprom/dep_prom/SiteAssets/Pages/

(Accessed: 10.01.2018).

7. Kantureeva Mansiya, Zakirova Alma (2014) The Methodology of Expert Systems. IJCSNS International Journal of Computer Science and Network Security, VOL.14 No.2, 2014. p. 6266

8. E.W.T. Ngai, S. Peng (2014) Decision support and intelligent systems in the textile and apparel supply chain: An academic review of research articles. Expert Systems with Applications 41 2014 p. 81-91

9. Pavlov M.A., Kirsanova E.A. (2015) Razrabotka fasetnoj klassifikacii materialov [Tekst] // Materialy dokladov 48 Mezhdunarodnoj nauchno-tehnicheskoj konferencii prepodavatelej i studentov, posvjashhennoj 50-letiju universiteta 2015. Tom 2 - p. 341-342.

10. Kirsanova E.A., Pavlov M.A., Demskaja A.A. (2016) Identifikacija jelementov baz dannyh i proizvodstvennyh zadach konfekcionirovanija materialov dlja zhenskih zhaketov // Dizajn i tehnologii. 2016. № 55 (97). p. 46-51

11. (2011) Al'tshuller G.Najti ideju: Vvedenie $v$ TRIZ - teoriju reshenija izobretatel'skih zadach / Genrih Al'tshuller. - 4-e izd. M.: Al'pina Pablisherz, 2011. - 400 p. 